\title{
BMJ Open Specchio-COVID19 cohort study: a longitudinal follow-up of SARS-CoV-2 serosurvey participants in the canton of Geneva, Switzerland
}

\author{
Helene Baysson (D) , ${ }^{1,2}$ Francesco Pennacchio, ${ }^{1}$ Ania Wisniak (D) ,1,2 \\ Maria Eugenia Zaballa, ${ }^{1}$ Nick Pullen, ${ }^{1}$ Prune Collombet, ${ }^{1,2}$ Elsa Lorthe, ${ }^{1}$ \\ Stéphane Joost (1D) , Jean-Francois Balavoine, ${ }^{4}$ Delphine Bachmann, ${ }^{5}$ \\ Andrew Azman, ${ }^{6,7}$ Didier Pittet, ${ }^{8}$ François Chappuis, ${ }^{9}$ Omar Kherad, ${ }^{10}$ \\ Laurent Kaiser, ${ }^{4,11,12}$ Idris Guessous, ${ }^{2,13}$ Silvia Stringhini, ${ }^{1,2,14}$ Specchio-COVID19 \\ study group
}

To cite: Baysson $\mathrm{H}$ Pennacchio F, Wisniak A, et al. Specchio-COVID19 cohort study: a longitudinal follow-up of SARS-CoV-2 serosurvey participants in the canton of Geneva, Switzerland. BMJ Open 2022;12:e055515. doi:10.1136/ bmjopen-2021-055515

- Prepublication history for this paper is available online To view these files, please visit the journal online (http://dx.doi. org/10.1136/bmjopen-2021055515).

IG and SS contributed equally.

Received 16 August 2021 Accepted 20 December 2021

Check for updates

(C) Author(s) (or their employer(s)) 2022. Re-use permitted under CC BY-NC. No commercial re-use. See rights and permissions. Published by BMJ.

For numbered affiliations see end of article.

Correspondence to Dr Helene Baysson; helene.baysson@unige.ch

\section{ABSTRACT}

Introduction The COVID-19 pandemic has affected billions of people around the world both directly through the infection itself and indirectly through its economic, social and sanitary impact. Collecting data over time is essential for the understanding of the disease spread, the incidence of COVID-19-like symptoms, the level and dynamics of immunity, as well as the long-term impact of the pandemic. The objective of the study was to set up a longitudinal follow-up of adult participants of serosurveys carried out in the canton of Geneva, Switzerland, during the COVID-19 pandemic. This followup aims at monitoring COVID-19 related symptoms and SARS-CoV-2 seroconversion, as well as the overall impact of the pandemic on several dimensions of health and on socioeconomic factors over a period of at least 2 years. Methods and analysis Serosurvey participants were invited to create an account on the dedicated digital platform Specchio-COVID19 (https://www.specchiocovid19.ch/). On registration, an initial questionnaire assessed sociodemographic and lifestyle characteristics (including housing conditions, physical activity, diet, alcohol and tobacco consumption), anthropometry, general health and experience related to COVID-19 (symptoms, COVID-19 test results, quarantines, hospitalisations). Weekly, participants were invited to fill in a short questionnaire with updates on self-reported COVID-19-compatible symptoms, SARS-CoV-2 infection testing and vaccination. A more detailed questionnaire about mental health, wellbeing, risk perception and changes in working conditions was proposed monthly. Supplementary questionnaires were proposed at regular intervals to assess more in depth the impact of the pandemic on physical and mental health, vaccination adherence, healthcare consumption and changes in health behaviours. At baseline, serology testing allowed to assess the spread of SARS-CoV-2 infection among the general population and subgroups of workers. Additionally, seropositive participants and a sample of randomly selected participants were invited for serologic testing at regular intervals in order to monitor both the seropersistance of anti-SARS-CoV-2 antibodies

\section{Strengths and limitations of this study}

- This is a large study with a diversified recruitment among the general population and mobilised workers, with at least half of the sample being based on random selection in the general population.

- A major strength of the study is the combined use of serological testing and questionnaires which will allow us to monitor the progression of the COVID-19 pandemic as well as to thoroughly analyse its effects on several dimensions of health.

- The longitudinal component of the study will provide insight into the extent and duration of immunity, as well as the long-term impact of the pandemic and the sanitary, social and economic measures associated with it.

- The main limitation is that Specchio-COVID19 is based on self-reported data with the exception of serological measures, which represents a risk of information bias.

- The study is primarily being conducted online, which may limit the generalisability of the findings, especially for the elderly and vulnerable populations, although internet access is extensive in Switzerland.

and the seroprevalence of anti-SARS-CoV-2 antibodies in the population of the canton of Geneva.

Ethics and dissemination The study was approved by the Cantonal Research Ethics Commission of Geneva, Switzerland (CCER Project ID 2020-00881). Results will be disseminated in a variety of ways, via the SpecchioCOVID-19 platform, social media posts, press releases and through regular scientific dissemination methods (openaccess articles, conferences).

\section{INTRODUCTION}

COVID-19, caused by infection with the novel coronavirus SARS-CoV-2, ${ }^{1}$ has impacted the lives and health of billions of people around 
the world. First reported in Wuhan, China, in December $2019,{ }^{2}$ the COVID-19 epidemic has since massively spread all over the globe, accounting for more than 131 million confirmed cases and more than 3 million deaths worldwide as of June, 2021. ${ }^{3}$ Due to the limited treatment options and and poor understanding of the virus itself, most governments have taken drastic measures to slow the epidemic spread by implementing massive population testing, quarantining of suspected cases and close contacts of infected individuals, isolation of confirmed cases, social distancing, shutting down of public infrastructures and other lockdown measures. ${ }^{4}$

These measures are expected to have a substantial long-term impact on the population's well-being, general and mental health and daily life, with a sudden disruption of professional, social or familial routines, as well as on national and local economies (including unemployment rates and working conditions). Psychological distress due to major uncertainties and stress related to the pandemic, as well as social isolation, may have an impact on mental health. ${ }^{5}$ Modified access to food, reduced travel and mobility, and a more sedentary lifestyle caused by the pandemic and related public health measures including lockdown and isolation/quarantine protocols, also have a strong potential for altering health behaviours, such as diet and physical activity. These changes may exacerbate in turn health and economic inequalities linked to demographic characteristics, socioeconomic status, residential area and social or clinical vulnerability, in particular for those with chronic diseases and/or mental health problems. Further, studies have shown that persistence of COVID-19 symptoms (mainly fatigue, dyspnoea and loss of taste or smell) can result in prolonged illness referred to as 'long COVID-19, ${ }^{, 7}$ or Post-Acute Sequelae of COVID-19, which might disproportionately impact vulnerable population groups. It is thus of utmost importance to monitor the potential long-term complications of SARS-CoV-2 infection ${ }^{67}$ as well as the consequences of potential postponement of medical interventions and screening programmes due to the pandemic.

Initial screening strategies for SARS-CoV-2 infection at the beginning of the pandemic often involved limiting tests to those with severe disease or risk factors for complications, thus failing to provide information on the full extent of the epidemic. One way to solve this issue has been to conduct population-based serosurveys, ${ }^{8}$ which provide estimates of the true proportion of the population having been exposed to the virus with or without symptoms. Only longer-term clinical and serological follow-up studies will allow us to evaluate the extent and duration of immunity, to assess risk factors for infection or re-infection, and to determine the frequency and risk factors for 'long COVID-19' symptoms among individuals who continue to report lasting effects long after being infected. ${ }^{6}$
Situation in the canton of Geneva and past serosurveys

The canton of Geneva, Switzerland, reported its first confirmed COVID-19 case on 26 February 2020, and by 22 June 2021, 58889 confirmed cases and 746 deaths had been reported. ${ }^{9}$ Since the beginning of April 2020, the Geneva University Hospitals and University of Geneva have conducted repeated cross-sectional seroprevalence surveys in representative samples of the population of the canton of Geneva. ${ }^{10}$ First, participants from the ongoing population-representative 'Bus Santé' study ${ }^{11}$ were invited to participate in the SEROCoV-POP serosurvey along with their household members aged 5 years and older. The study was carried out over a total of 12 weeks (April-June 2020) and included 8344 participants. ${ }^{10}$ A second serosurvey, SEROCoV-WORK+ (MaySeptember 2020), recruited 10582 essential workers who could not telework during the confinement period associated with the first epidemic wave. ${ }^{12}$ To determine population seroprevalence during the second epidemic wave, a third serosurvey was conducted during November 2020 in the framework of Corona Immunitas, a national research programme assessing the seroprevalence of antiSARS-CoV-2 antibodies across Switzerland. ${ }^{13}$ In order to be more representative of some age groups, children and their family, as well as people over 65 years were randomly selected from population registries and invited to perform serological testing. A random sample of participants from the general population who had participated in the serosurvey of spring 2020 was also invited for a second assessment. Results showed that the prevalence of anti-SARS-CoV-2 antibodies was around $11 \%$ in Geneva in May $20200^{10}$ and $21 \%$ by December $2020 .{ }^{14}$ Additional serosurveys will be conducted during the follow-up period in order to obtain updated seroprevalence estimates for the general population of the canton of Geneva.

In this context, our objective was to set up a longitudinal cohort study for the long-term monitoring of adult participants from all aforementioned serosurveys. This follow-up will take place through the Specchio-COVID19 digital platform and will monitor COVID-19-related symptoms and SARS-CoV-2 seroconversion, as well as the overall impact of the pandemic on several dimensions of health and on socioeconomic factors over a period of at least 2 years.

\section{METHODS/DESIGN}

Follow-up of serosurvey participants using the SpecchioCOVID19 digital platform will allow for the serosurveillance of the COVID-19 epidemic in Geneva as well as the continuous and dynamic monitoring of the population's physical and mental health through online questionnaires and through repeated serological testing (figure 1). More specifically, the Specchio-COVID19 study, using a dedicated digital platform, aims to:

- Monitor the epidemic and infection propagation among the general population of the canton of Geneva and among selected subpopulations 


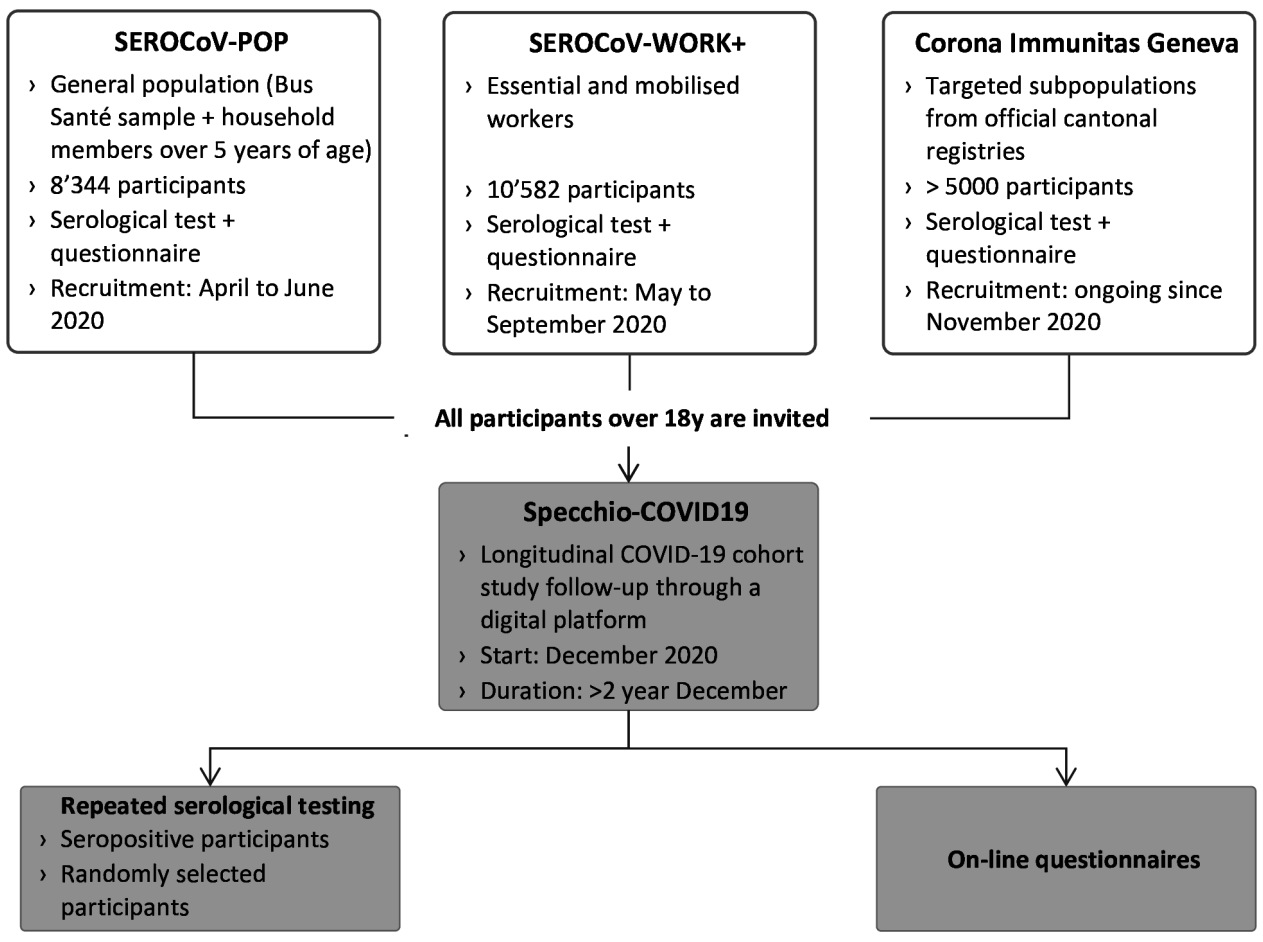

Figure 1 Design of Specchio-COVID19 study.

depending on their sociodemographic characteristics (age), occupationnal (eg, mobilised workers vs others workers) or clinical (eg, participants with chronic diseases).

- Identify risk factors and potentially protective factors for infection.

- Evaluate the persistence of anti-SARS-CoV-2 antibodies over time and the probability of reinfection.

- Evaluate the overall health impact of the COVID-19 pandemic in the short and long term across a large number of dimensions, including physical and mental health, behavioural changes (diet, physical activity, tobacco and alcohol consumption), forgoing healthcare, incidence of non-communicable diseases in the population, and persistence of COVID-19-related symptoms ('long COVID-19'). This evaluation will be carried out by comparison between those diagnosed with COVID-19 to others. Data will also be compared with observations relating to the prepandemic period (Bus sante Study). ${ }^{11}$

- Evaluate the overall socioeconomic impact on the population of the COVID-19 pandemic, including in the long term; using data in Switzerland for the prepandemic period.

- Evaluate risk perception, the adoption of preventive behaviours and acceptance of COVID-19-related public health policies over time.

- Collaborate as part of the Corona Immunitas national research programme (https://www.corona-immunitas.ch/), coordinated by the Swiss School of Public Health $(\mathrm{SSPH}+)$ which aims to generate comparable seroprevalence estimates across Switzerland. ${ }^{13}$

\section{Study design}

In order to quickly respond to the COVID-19-related health emergency, and thus support public health actors in decision making, we decided to take advantage of an online digital platform of the population of Geneva (Specchio) developed through a collaboration between the University of Geneva and the Health Directorate of the canton of Geneva. ${ }^{15}$ The Specchio-COVID-19 internet platform allows prospective longitudinal follow-up of participants of serosurveys in the canton, resulting in the constitution of the Specchio-COVID19 digital cohort. The Specchio-COVID19 internet platform was launched in November 2020. The Specchio-COVID19 cohort will be followed up for at least 2 years (November 2022).

\section{Recruitment and inclusion}

Participants of the Specchio-COVID19 platform are currently being recruited among adult participants of serosurveys conducted in Geneva (figure 1). Progressively, we plan to extend the recruitment to other targeted populations in Geneva. All participants over the age of 18 for whom an email address is on file receive an invitation to create a personal account on the Specchio-COVID19 digital platform. Informed consent is obtained during the baseline serology testing visit.

On registration on the digital platform (figure 2), participants are included in the study and then followed via a website specifically created for this purpose (figure 3). Questionnaires can be directly filled-in online using a secure HyperText Markup Language (HTML) interface, where all requirements to guarantee the secured management and storage of sensitive data 


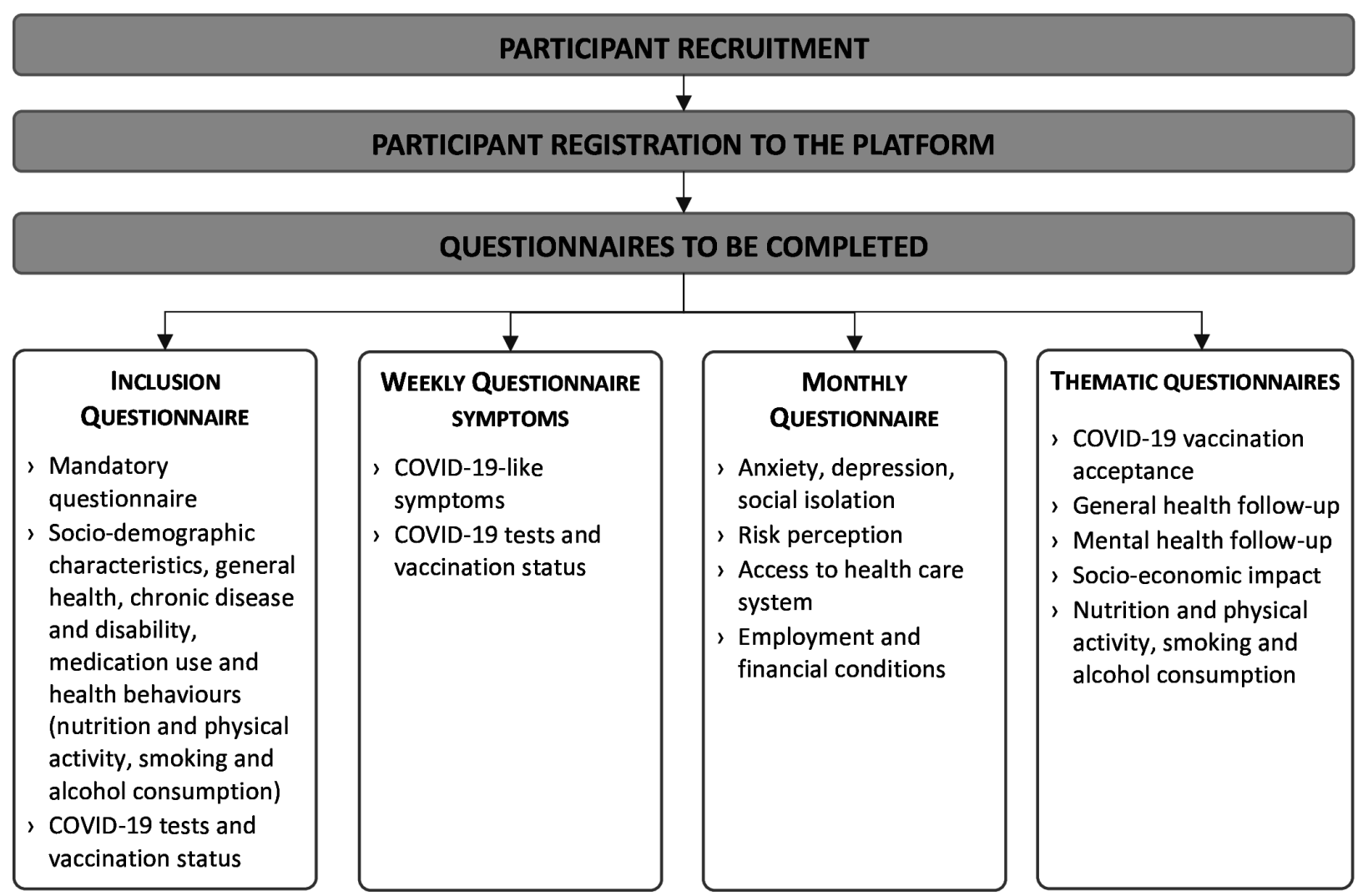

Figure 2 Recruitment and questionnaires.

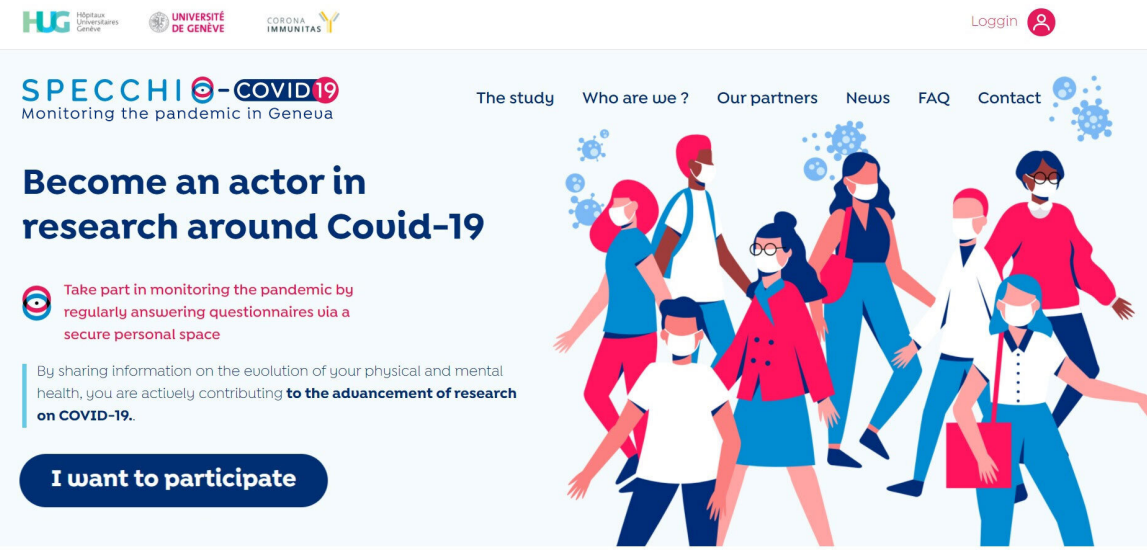

How does it work?

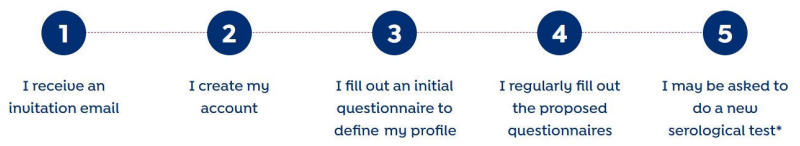

(2) "Depending on your answers, and according to the needs of the study, a new serological test (assay of anti-SARS-CoV-2 antibodies) may be offered to you

- Specchio-CoviD19 - Note d'information et politique de confidentialité

Figure 3 Home page of Specchio-COVID19 platform translated from French, www.specchio-COVID19.ch (only available in French at the time of submission). 
are met. In order to be included in the cohort, participants have to fill in a mandatory initial questionnaire. After this inclusion step, other questionnaires related to the COVID-19 pandemic and its impact on physical and mental health and well-being are proposed to participants on a regular basis (figure 2). The content of these questionnaires is detailed below in sections 'Digital Follow-up' and 'Additional questionnaires'. Serological tests were performed on all participants at baseline and are repeated over time for subgroups of participants, selected according to the needs of the study at each stage (see below).

\section{Data collection techniques \\ Information collected at inclusion}

At inclusion, via an initial questionnaire completed on-line by the participant, information is collected on sociodemographic characteristics, general heath, presence of chronic diseases and physical or mental disability, medication use and health behaviours (diet, physical activity, alcohol and tobacco consumption, forgoing healthcare). Regarding sociodemographic characteristics, information is collected regarding residential postal code, household composition (number of cohabitants, number of children) and housing conditions, work situation (employment status, profession), education level and income. As the canton of Geneva is cosmopolitan with $40 \%$ of residents and workers of foreign origin, ${ }^{16}$ additional information is collected about nationality, ethnicity and mother tongue. Subjective health status (perceived general health and perceived mental health), current weight, vaccination status and pregnancy status are also collected at inclusion.

\section{Biological and clinical follow-up}

Follow-up of seropositive participants

Seropositive participants are invited for serological testing at regular intervals (approximatively every 4 months), regardless of their symptomatology. This allows us to monitor the persistence of anti-SARS-CoV-2 antibodies over time. A first seropersistence survey was conducted in November 2020, showing seropersistence of anti-SARSCoV-2 antibodies in $100 \%$ of participants at 115-224 days after their baseline serological test, as measured by the Elecsys anti-RBD assay (Roche Diagnostics, Rotkreuz, Switzerland). ${ }^{16}$

\section{Follow-up of randomly selected participants}

Depending on the evolution of the COVID-19 pandemic in Geneva and in coordination with the Corona Immunitas national research programme, samples of randomly selected participants from population registries and among our longitudinal cohort participants are repeatedly invited for serological testing, regardless of their symptomatology or previous serological status, in order to monitor the seroprevalence of anti-SARS-CoV-2 antibodies in the general population over time.

\section{Serological testing}

Assays used for serological testing may vary during the course of the study due to new, more performant tests being commercialised. At baseline or follow-up visits of our study, SARS-CoV-2 antibodies have been measured using three commercially-available tests: a semiquantitative anti-S1 ELISA detecting IgG (Euroimmun, Lübeck, Germany \#EI 2606-9601 G, referred to as EI), and the quantitative Elecsys anti-RBD (\#09 289275 190, Roche-S) and semiquantitative Elecsys anti-N (\#09 203079 190, Roche-N), both measuring IgG/A/M levels (Roche Diagnostics, Rotkreuz, Switzerland). The assessment of antibodies against the spike protein (anti-S) and the nucleocapsid protein (anti-N) of SARS-CoV-2 permits, in principle, to identify individuals with past infection even if they were vaccinated against COVID-19 (as mRNA-based vaccines are the only ones so far approved in Switzerland). All serological tests are centralised and performed blinded to participants' characteristics or clinical history.

\section{Digital follow-up}

The digital follow-up consists of brief weekly updates with respect to self-reported symptoms compatible with COVID-19, SARS-CoV-2 testing (RT-PCR or serological tests), and vaccination status. In a more detailed monthly follow-up, questions are addressed about general and mental health, well-being and risk perception, changes in health behaviours (diet, physical activity, alcohol and tobacco consumption, sleep quality) and changes in work conditions or employment. Regarding mental health, Patient Health Questionnaire 2-items (PHQ-2), ${ }^{17}$ Generalized Anxiety Disorder 2-item (GAD-2) ${ }^{18}$ and K6 ${ }^{19}$ (19) scores are used to assess respectively depression anxiety and psychologic distress. Questions about social isolation and feelings of loneliness are also asked.

\section{Additional questionnaires}

General and mental health (assessed respectively every 3 and 6 months)

Questions about long-term COVID-19-related symptoms are addressed in order to better characterise long COVID-19 illness. COVID-19 vaccination status and dates of doses were recorded as well as new health events (newly diagnosed chronic diseases, new treatments, hospitalisations) using mandatory questions

\section{Family life and children (one time questionnaire)}

Participants who indicate having children under 18 are invited to answer additional questions about their children's stress levels and their parental experiences during the pandemic (in line with home schooling and school closures).

\section{Access to healthcare (one time questionnaire)}

Specific questionnaires will assess the effects of the COVID-19 pandemic on the utilisation of health and social care services, and medication adherence. 
Employment and working conditions (one time questionnaire) A specific questionnaire will be developed on employment status, working conditions and financial status to get an overview of the economic impact of the pandemic in the population of Geneva, including subgroups of essential workers who could not telework during (semi) lockdown periods.

Vaccination (one time questionnaire)

A specific questionnaire assesses acceptability and uptake of the vaccine against SARS-CoV-2.

\section{Participants and public involvement}

Given the high frequency of questionnaires to be filled in and the long duration of the follow-up period, several measures were designed to maintain high retention of participants. These measures include regular electronic newsletters with links to the 'News' Specchio-COVID19 webpage, containing short videos describing the study and novel findings, media releases and accessible summaries of scientific articles published as part of the Specchio-COVID19 project. The organisation of webinars specifically dedicated to participants is also planned. These activities are specifically designed to inform the participants and motivate them to remain in the study. Regarding participant involvement, questionnaires include open comment fields in which participants can describe their experiences related to the pandemic or to the study questionnaires. A specific email address and a dedicated hotline were set up so that participants can get in touch with the Specchio-COVID19 team. Participants receive personal answers in case of technical difficulties or any questions about the study or their serological results. Participants' comments are considered when improving revised versions of the questionnaires. Other examples of participant communication include result visualisations on the national research programme Corona-Immunitas website (https://www.corona-immunitas.ch/), coordinated by the $\mathrm{SSPH}+$ and short videos on the CoronaImmunitas' YouTube channel (eg, Science in A Minute, ...), which are regularly updated.

\section{Statistical analysis plan}

We plan to use this study to conduct timely data collection and analyses meeting public health or scientific needs. In this context, new scientific questions may arise regarding the impact of the pandemic and the evolving scientific knowledge about SARS-CoV-2 transmission. When new questions will arise, we will develop analysis plans prior to collecting data through new questionnaires and biological testing and undertaking analyses. The data collected will be analysed for the entire study population and for subgroups defined by demographics, comorbidities or clinical characteristics.

Through the serosurveys conducted in the canton of Geneva and the national research programme Corona Immunitas, we anticipate enrolling at least 10000 participants. The following section provides an overview of 'basic' analytical strategies that will be conducted with the data. Entered data will be summarised first by using simple descriptive statistics. Then, appropriate statistical methods will be used, especially $\chi^{2}$ tests and general linear models. Bayesian regression models will be performed for estimating population seroprevalence by accounting for age, sex and test performance. Analyses will be conducted in $\mathrm{R}$ ( $\mathrm{R}$ Foundation for Statistical Computing, Vienna, Austria) or STATA (StataCorp) environments.

\section{Ethics and dissemination}

\section{Ethical considerations}

The purpose of this study is explained to all individuals at the time of their first serological testing performed in the context of one of the serosurveys, all approved by the Cantonal Research Ethics Commission of Geneva, Switzerland (CCER project ID 2020-00881 and PB_2016-00363). Informed signed consent is obtained for participation in the Specchio-COVID19 study. Personal identifying information is stored in a password-protected database containing only contact details. The data from questionnaire responses and biological results are stored in a separate password-protected anonymous SUGAR-CRM database (https://www.sugarcrm.com). Only the administrators have access to the identity of the participants, in order to organise visits for biological testing. The data is stored on secure servers in Switzerland for as long as they are needed to achieve the study objectives. Compliance with data protection regulations was approved by the Cantonal Research Ethics Commission of Geneva, as part of the SEROCoV-WORK + study protocol (CCER Project ID 2020-00881).

\section{Dissemination}

Due to the evolving nature of the COVID-19 pandemic, results are disseminated in a variety of ways. As mentioned earlier, results are communicated through the SpecchioCOVID19 platform via dedicated webpages ('News' page, 'Research' pages) and via an electronic newsletter which is regularly sent by email to participants and collaborators. Where appropriate, press releases and social media are targeted. Results are also released through traditional scientific dissemination methods, journal articles, open-access publications and conference presentations. Of note, the Specchio-COVID19 study, integrated in the Corona Immunitas national research programme, also provides data to the Swiss Federal Office of Public Health and to the Swiss COVID-19 scientific task force addressing key questions concerning seroprevalence of anti-SARSCoV-2 antibodies, population adherence to preventive measures (facemasks, social distancing) and COVID-19 vaccine uptake.

\section{DISCUSSION}

Thanks to the Specchio-COVID19 platform, we will continue to monitor the evolution of anti-SARS-CoV-2 seroprevalence in the population of the canton of Geneva. 
Longer-term clinical and serological follow-up via the Specchio-COVID-19 platform will allow us to monitor the duration of anti-SARS-CoV-2 antibodies and the possible clinical sequelae of COVID-19 over time. Data collected via Specchio-COVID19 will also permit more detailed analyses on the seroprevalence distribution within the population, taking into account symptomatology and sociodemographic factors, to better understand transmission and selective risk of infection within the community. Immunity dynamics related to anti-SARS-CoV-2 antibodies are still largely unknown. For example, whether the presence of anti-SARS-CoV-2 antibodies protects individuals from a new COVID-19 infection, especially in relation to the circulation of new variants, is unknown at this stage. If it does, the duration of immunity also needs to be evaluated. The duration of seroprotection of vaccinated individuals also remains unknown at the population level. Following a large and diversified cohort, with blood samples collected and stored for serological analyses since the beginning of the pandemic, will allow us to address these questions.

Detailed information on the effects of the pandemic on mental and physical health, as well as on social and professional life are also collected. Although monitoring of COVID-19 patients and contact-tracing is assured by the official state department in charge, independently from our study, expected results will help design adequate public health policies to manage new potential outbreaks of the COVID-19 pandemic but also to face the possible clinical complications that may occur after the acute phase of the disease subsides (long COVID-19) and to prepare the healthcare system for such challenges. The long-term implications of SARS-CoV-2 infection on morbidity and mortality are as of yet unknown, although some basic deductions can be made based on clinical experience gained from residual long-term effects of SARS and MERS epidemics. ${ }^{20}$ Long-term complications need to be carefully followed up over time. Moreover, indirect long-term effects of public health measures on health, such as the effects of social distancing on mental health or the consequences of healthcare renunciation on chronic disease management, are also expected.

\section{Strengths}

Strengths of our project pertain to its large sample size and recruitment of participants across different populations (general population, non-confined workers). Around 20000 participants already included in either one of the SEROCoV-POP, SEROCoV-WORK+ and Corona Immunitas Geneva serosurveys have been invited by email to join the Specchio-COVID19 platform at the end of 2020. Assuming a participation rate of $50 \%$, we estimate that around 10000 participants will be included by the end of 2021, leading to a cohort of participants that will be followed over time. It is also planned to carry out a specific study focusing on children (SEROCoVKIDS), who will be integrated in to our digital cohort, and to open the recruitment more extensively through time, depending on scientific needs. The flexibility of the Specchio-COVID-19 web platform allows for a quick implementation of new questionnaires to provide insights into multiple aspects of this singular situation at a largescale level, while adapting to the evolving phases of the pandemic. Previous initiatives in Switzerland, such as Grippenet, ${ }^{21}$ have demonstrated the potential for using online surveys for disease surveillance. ${ }^{22}$ Another strength of the Specchio-COVID19 project is the collaboration of researchers across a wide range of disciplines (epidemiologists, infectious diseases specialists, medical doctors, psychologists, biologists, (data scientists) and statisticians) in designing and implementing questionnaires, enabling a broad spectrum of research questions to be addressed. One of the major strengths is that Specchio-COVID19 combines data from self-reported questionnaires and results from biological testing. Self-reported data on socioeconomic characteristics, symptoms and contact tracing provides understanding of the pandemic in more depth than serological testing alone. It will enable assessment of the severity of illness more accurately, as well as transmission dynamics, and the effect of socioeconomic characteristics.

As a subsample of the participants of SpecchioCOVID19 have also been included in the 'Bus Santé' study, ${ }^{11}$ an annual health survey of a representative sample of the population of the canton of Geneva which has been carried out for almost 30 years, it is possible to compare data collected through paper questionnaires and face-to-face clinical interviews (in the 'Bus Santé' study) with data collected through online questionnaires (via the Specchio-COVID19 platform). This will allow us to provide an insight into declaration bias for social desirability answers (weight, tobacco consumption, etc) and to acquire data on behavioural changes before and after the pandemic for a subsample of our participants.

\section{Limitations}

Some limitations should be acknowledged. SpecchioCOVID19 is based on voluntary involvement. Reasons why individuals might not participate include lack of time and motivation, poor internet access, language restrictions or a poor understanding of the purpose of the study. As in previous studies with a comparable design,${ }^{23}$ it is expected that more women than men and highly educated participants with a high socioeconomic status will participate in comparison with the general population of the canton of Geneva. To increase participation rate among socially vulnerable or less educated populations, targeted recruitment will be carried out with the support of associations in Geneva. In the future, depending on our financial resources, it is planned to have a dedicated team, ready to provide help in filling on-line questionnaires Web-based participation might exclude less technologyproficient individuals or people with no internet access. To mitigate this limitation, it is planned to send paper questionnaires at less frequent intervals (every 6 months approximatively) to individuals who make such a request 
at their visit. With time, we will provide study documents in languages other than French and provide a multilanguage platform. To minimise social desirability bias (eg, regarding health preventive behaviours), questionnaires are web-based and anonymity is guaranteed. Finally, we plan to use our original platform Specchio ${ }^{15}$ after the pandemic ends to continue following participants of Specchio-COVID19 for the coming decade and beyond, providing opportunities to examine the long-term health impacts of the pandemic.

As for any other long-term prospective cohort, attrition remains a major concern. Over time, willingness to participate to the study may diminish considerably and low response rates might affect the representativeness of the study population, the generalisability of the findings, and the power of the statistical analyses. In order to favour its use and maintain participation over time, the online platform Specchio-COVID-19 is designed to be attractive and user-friendly. Email reminders will be sent to encourage participation, and in case of non-response or incomplete questionnaires. Motivational strategies will be implemented to improve retention of participants, for example, 'digital awards' (eg, a trophy sticker) or participant certificates. Communication with participants will be reinforced through regular updates on the progress and main achievements of the project via the SpecchioCOVID19 platform and a periodic newsletter. A team specifically trained and dedicated to Specchio-COVID-19 informs and responds to participants' queries. As the research topic is of utmost interest for many people in the current situation of a pandemic, the response rate is expected to be higher in the Specchio-COVID19 study than in other non-COVID-19-related studies. However, one key issue is that participants will be aware of their serological status during follow-up, which may affect behaviour and risk perception, and therefore willingness to participate in the study over time.

\section{Links to other national and international research programmes}

In order to have a broader overview of the consequences of the pandemic beyond Geneva, Specchio-COVID19 collaborates with the Corona Immunitas national research, coordinated by the SSPH+. This national programm aims to harmonise ongoing seroprevalence studies across Switzerland, targeting the population as a whole or particular subgroups, such as children, seniors, health professionals, essential and non-confinable workers, or vulnerable populations. Comparable results of these studies will provide an accurate view of the proportion of the Swiss population infected with the virus and of the impact of the pandemic on several dimensions of health. ${ }^{13}$ Comparisons with international projects carried out in several European countries are possible, and will provide unique opportunities to examine the effect of different governmental measures on seroprevalence, mental health and well-being of the population and on the economical and societal impact of the pandemic worldwide. In
Germany, the KoCo19 (prospective COVID-19 cohort) study included more than 5000 participants drawn from a random sample of 3000 households in Munich. ${ }^{24}$ After a baseline visit conducted between April and June 2020, participants completed an online household questionnaire and an online personal questionnaire, a daily digital diary about symptoms, outings, use of public transportation, social contacts as well as additional questions about the psychosocial and economic situation. In France, the Sapris-SERO study was implemented in March 2020 to evaluate the main epidemiological, social and behavioural challenges of the SARS-CoV2 epidemic in France in relation to social inequalities in health and healthcare. It is based on a consortium of 16000 participants selected from ongoing prospective cohort studies including three general population-based adult cohorts and two child cohorts. ${ }^{25}$ In Luxembourg, 1862 participants were followed since April 2020 as part of the CONVINCE study, a national survey of randomly selected asymptomatic adults. ${ }^{26}$

\section{Author affiliations}

${ }^{1}$ Unit of Population Epidemiology, Division of Primary Care Medicine, Geneva University Hospitals, Geneva, Switzerland

${ }^{2}$ Department of Health and Community Medicine, Faculty of Medicine, University of Geneva, Geneva, Switzerland

${ }^{3}$ Laboratory of Geographic Information Systems (LASIG), School of Architecture, Civil and Environmental engineering (ENAC), Ecole Polytechnique Fédérale de Lausanne (EPFL), Lausanne, Switzerland

${ }^{4}$ Department of Medicine, Faculty of Medicine, University of Geneva, Geneva,

Switzerland

${ }^{5}$ Hirslanden Clinique des Grangettes and Hislanden Clinique La Colline, Geneva, Switzerland

${ }^{6}$ Department of Epidemiology, Johns Hopkins Bloomberg School of Public Health, Baltimore, MD, USA

${ }^{7}$ Institute of Global Health, Faculty of Medicine, University of Geneva, Geneva, Switzerland

${ }^{8}$ Infection Control Program and World Health Organization Collaborating Center on Patient Safety, Geneva University Hospitals and Faculty of Medecine, University of Geneva, Geneva, Switzerland

${ }^{9}$ Department of Primary Care Medicine, Geneva University Hospitals, Geneva, Geneva, Switzerland

${ }^{10}$ Division of Internal Medicine, Hôpital de la Tour and Faculty of Medicine, University of Geneva, Geneva, Switzerland

${ }^{11}$ Geneva Center of Emerging Viral Diseases and Laboratory of Virology, Geneva University Hospitals, Geneva, Switzerland

${ }^{12}$ Division of Laboratory Medicine, Geneva University Hospitals, Geneva, Switzerland

${ }^{13}$ Division of Primary Care Medicine, Geneva University Hospitals, Geneva, Switzerland

${ }^{14}$ University Center for General Medicine and Public Health, University of Lausanne, Lausanne, Switzerland

Correction notice This article has been corrected since it was first published. The name of the authors has been updated.

Acknowledgements The authors are indebted to the participants of the SpecchioCOVID19 cohort for their commitment and cooperation. This study would not have been possible without the instrumental and passionate contribution of the staff of the Unit of Population Epidemiology of the Primary Care Division, Geneva University Hospitals, Geneva, Switzerland and the professionalism of our partners Med\&Hyg and DotBASE, and all our colleagues not included in the Specchio-COVID19 study group. We particularly thank the Specchio-COVID19 operational team who spend a dedicated time responding to participants' queries.

Collaborators Specchio-COVID19 study group: Isabelle Arm-Vernez, Andrew S Azman, Fatim Ba, Oumar Ba, Delphine Bachmann, Jean-François Balavoine, 
Michael Balavoine, Hélène Baysson, Lison Beigbeder, Julie Berthelot, Patrick Bleich, Gaëlle Bryand Rumley, François Chappuis, Prune Collombet, Delphine Courvoisier, Alain Cudet, Carlos de Mestral Vargas, Paola D'Ippolito, Richard Dubos, Roxane Dumont, Isabella Eckerle, Nacira El Merjani, Antoine Flahault, Natalie Francioli, Marion Frangville, Idris Guessous, Séverine Harnal, Samia Hurst, Laurent Kaiser, Omar Kherad, Julien Lamour, Pierre Lescuyer, François L'Huissier, Fanny-Blanche Lombard, Andrea Jutta Loizeau, Elsa Lorthe, Chantal Martinez, Lucie Ménard, Lakshmi Menon, Ludovic Metral-Boffod, Benjamin Meyer, Alexandre Moulin, Mayssam Nehme, Natacha Noël, Francesco Pennacchio, Javier Perez-Saez, Giovanni Piumatti, Didier Pittet, Jane Portier, Klara M Posfay-Barbe, Géraldine Poulain, Caroline Pugin, Nick Pullen, Zo Francia Randrianandrasana, Aude Richard, Viviane Richard, Frederic Rinaldi, Jessica Rizzo, Khadija Samir, Claire Semaani, Silvia Stringhini, Stéphanie Testini, Didier Trono, Guillemette Violot, Nicolas Vuilleumier, Ania Wisniak, Sabine Yerly, María-Eugenia Zaballa.

Contributors SS, IG, HB, FP, AW, MEZ, NP, PC, EL, SJ, J-FB, DB, AA, DP, FC, OK and LK, designed the study. HB, AW, MEZ and SS wrote the article. HB, FP, AW, MEZ, NP, PC, EL, SJ, J-FB, DB, AA, DP, FC, OK, LK, IG and SS commented on drafts of paper and approved its final version.

Funding This study was funded by the Swiss Federal Office of Public Health, the General Directorate of Health of the Department of Safety, Employment and Health of the canton of Geneva, the Private Foundation of the Geneva University Hospitals, the Swiss School of Public Health (Corona Immunitas Research Programme) and the Fondation des Grangettes. Award/Grant number are not applicable.

Competing interests None declared.

Patient and public involvement Patients and/or the public were involved in the design, or conduct, or reporting, or dissemination plans of this research. Refer to the Methods section for further details.

Patient consent for publication Not applicable.

Provenance and peer review Not commissioned; externally peer reviewed.

Open access This is an open access article distributed in accordance with the Creative Commons Attribution Non Commercial (CC BY-NC 4.0) license, which permits others to distribute, remix, adapt, build upon this work non-commercially, and license their derivative works on different terms, provided the original work is properly cited, appropriate credit is given, any changes made indicated, and the use is non-commercial. See: http://creativecommons.org/licenses/by-nc/4.0/.

\section{ORCID IDs}

Helene Baysson http://orcid.org/0000-0003-0916-6958

Ania Wisniak http://orcid.org/0000-0002-3942-2134

Stéphane Joost http://orcid.org/0000-0002-1184-7501

\section{REFERENCES}

1 Coronaviridae Study Group of the International Committee on Taxonomy of Viruses. The species severe acute respiratory syndrome-related coronavirus: classifying 2019-nCoV and naming it SARS-CoV-2. Nat Microbiol 2020;5:536-44.

2 Zhu N, Zhang D, Wang W, et al. A novel coronavirus from patients with pneumonia in China, 2019. N Engl J Med 2020;382:727-33.

3 World Health organization (who), 2019. Available: https://www.who. int/fr/emergencies/diseases/novel-coronavirus-2019

4 Ayouni I, Maatoug J, Dhouib W, et al. Effective public health measures to mitigate the spread of COVID-19: a systematic review. BMC Public Health 2021;21:1015.

5 Brooks SK, Webster RK, Smith LE, et al. The psychological impact of quarantine and how to reduce it: rapid review of the evidence. The Lancet 2020;395:912-20.
6 Mahase E. Covid-19: What do we know about "long covid"? BMJ 2020;370:m2815.

7 Nehme M, Braillard O, Alcoba G, et al. COVID-19 symptoms: longitudinal evolution and persistence in outpatient settings. Ann Intern Med 2021;174:723-5.

8 Lai C-C, Wang J-H, Hsueh P-R. Population-based seroprevalence surveys of anti-SARS-CoV-2 antibody: an up-to-date review. Int $J$ Infect Dis 2020;101:314-22.

9 République et Canton de Genève. Données cantonales. Available: https://infocovid.smc.unige.ch/ [Accessed 17 Jan 2021].

10 Stringhini S, Wisniak A, Piumatti G, et al. Seroprevalence of antiSARS-CoV-2 IgG antibodies in Geneva, Switzerland (SEROCoVPOP): a population-based study. Lancet 2020;396:313-9.

11 Guessous I, Bochud M, Theler J-M, et al. 1999-2009 trends in prevalence, unawareness, treatment and control of hypertension in Geneva, Switzerland. PLoS One 2012;7:e39877.

12 Stringhini S, Zaballa M-E, Pullen N, et al. Large variation in antiSARS-CoV-2 antibody prevalence among essential workers in Geneva, Switzerland. Nat Commun 2021;12:3455.

13 West EA, Anker D, Amati R, et al. Corona Immunitas: study protocol of a nationwide program of SARS-CoV-2 seroprevalence and seroepidemiologic studies in Switzerland. Int J Public Health 2020;65:1529-48.

14 Stringhini S, Zaballa M-E, Perez-Saez J, et al. Seroprevalence of antiSARS-CoV-2 antibodies after the second pandemic peak. Lancet Infect Dis 2021;21:600-1.

15 Baysson $\mathrm{H}$, Joost S, Attar Cohen $\mathrm{H}$, et al. Development of a webplatform for dynamic monitoring of population health in Geneva: Specchio project. 16th world Congress on public health 2020, 15-19 October 2020 (on line). Eur J Public Health 2020;30.

16 Perez-Saez J, Zaballa M-E, Yerly S, et al. Persistence of anti-SARS-CoV-2 antibodies: immunoassay heterogeneity and implications for serosurveillance. Clin Microbiol Infect 2021;27:2021.03.16.21253710.

17 Arthurs E, Steele RJ, Hudson M, et al. Are scores on English and French versions of the PHQ-9 comparable? an assessment of differential item functioning. PLoS One 2012;7:e52028.

18 Spitzer RL, Kroenke K, Williams JBW, et al. A brief measure for assessing generalized anxiety disorder: the GAD-7. Arch Intern Med 2006;166:1092-7.

19 Staples LG, Dear BF, Gandy M, et al. Psychometric properties and clinical utility of brief measures of depression, anxiety, and general distress: the PHQ-2, GAD-2, and K-6. Gen Hosp Psychiatry 2019;56:13-18

20 O'Sullivan O. Long-Term sequelae following previous coronavirus epidemics. Clin Med 2021;21:e68-70.

21 Richard A, Müller L, Wisniak A, et al. Grippenet: a new tool for the monitoring, risk-factor and vaccination coverage analysis of influenza-like illness in Switzerland. Vaccines 2020;8:343.

22 Ming LC, Untong N, Aliudin NA, et al. Mobile health Apps on COVID-19 Launched in the early days of the pandemic: content analysis and review. JMIR Mhealth Uhealth 2020;8:e19796.

23 Hercberg S, Castetbon K, Czernichow S, et al. The Nutrinet-Santé study: a web-based prospective study on the relationship between nutrition and health and determinants of dietary patterns and nutritional status. BMC Public Health 2010;10:242.

24 Radon K, Saathoff E, Pritsch M, et al. Protocol of a population-based prospective COVID-19 cohort study Munich, Germany (KoCo19). BMC Public Health 2020;20:1036.

25 Carrat F, de Lamballerie X, Rahib D. Seroprevalence of SARS-CoV-2 among adults in three regions of France following the lockdown and associated risk factors: a multicohort study. Int J Epidemiol. 2021 Nov2020;10:5:1458-78. doi:10.1093/ije/dyab110

26 Snoeck CJ, Vaillant M, Abdelrahman T. Prevalence of SARS-CoV-2 infection in the Luxembourgish population - the CON-VINCE study. medRxiv 2020;2020. 